\title{
EXPONENTIAL FACTORIZATIONS OF HOLOMORPHIC MAPS
}

\author{
FRANK KUTZSCHEBAUCH AND LUCA STUDER
}

\begin{abstract}
We show that any element of the special linear group $\mathrm{SL}_{2}(\mathrm{R})$ is a product of two exponentials if the ring $\mathrm{R}$ is either the ring of holomorphic functions on an open Riemann surface or the disc algebra. This is sharp: one exponential factor is not enough since the exponential map corresponding to $\mathrm{SL}_{2}(\mathbb{C})$ is not surjective. Our result extends to the linear group $\mathrm{GL}_{2}(\mathrm{R})$.
\end{abstract}

\section{INTRODUCTION}

For a Stein space $X$, a complex Lie group $G$ and its exponential map $\exp : \mathfrak{g} \rightarrow G$ we say that a holomorphic map $f: X \rightarrow G$ is a product of $k$ exponentials if there are holomorphic maps $f_{1}, \ldots, f_{k}: X \rightarrow \mathfrak{g}$ such that

$$
f=\exp \left(f_{1}\right) \cdots \exp \left(f_{k}\right) .
$$

It is easy to see that any map $f$ which is a product of exponentials (for some sufficiently large $k$ ) is null-homotopic. In the case where $G$ is the special linear group $\mathrm{SL}_{n}(\mathbb{C})$ the converse follows from [6] as explained in [1]. However, it turns out to be a difficult problem to determine the minimal number $k$ of needed factors in dependence of the dimensions of $X$ and $\mathrm{SL}_{n}(\mathbb{C})$. We solve this problem for $\operatorname{dim} X=1$ and $n=2$.

Theorem 1. Any holomorphic map from an open Riemann surface to the special linear group $\mathrm{SL}_{2}(\mathbb{C})$ is a product of two exponentials.

Theorem 1improves a result of Doubtsov and Kutzschebauch, who showed the same result with three instead of two factors in the conclusion, see Proposition 3 in [1. Stated differently, Theorem 1 1 says that every element of $\mathrm{SL}_{2}(\mathcal{O}(X))$ can be written as a product of two exponentials, where $\mathcal{O}(X)$ denotes the ring of holomorphic functions on a given open Riemann surface $X$. Our second result is of similar flavor, but the $\operatorname{ring} \mathcal{O}(X)$ is replaced by the disc algebra $\mathcal{A}$. By definition, the disc algebra $\mathcal{A}$ is the $\mathbb{C}$-Banach algebra of continuous functions on the closed disc $\{z \in \mathbb{C}:|z| \leq 1\}$ which are holomorphic on the interior, equipped with the supremum norm.

Theorem 2. For the disc algebra $\mathcal{A}$, any element of $\mathrm{SL}_{2}(\mathcal{A})$ is a product of two exponentials.

1991 Mathematics Subject Classification. 15A54, 15A16, 30H50, 32A38, 32E10, 48E25.

Key words and phrases. products of exponentials, Bass stable rank, rings of holomorphic functions.

The research of the first author was partially supported by Schweizerische Nationalfonds Grant 200021-178730. The research of the second author was partially supported by Schweizerische Nationalfonds Grant PP00P2_157583. 
Recall that the exponential map exp $: \mathfrak{s l}_{2}(\mathbb{C}) \rightarrow \mathrm{SL}_{2}(\mathbb{C})$ is not surjective. In this sense Theorem 1 and 2 are sharp. It is worth mentioning that $\mathrm{SL}_{2}(\mathbb{C})$ is simply connected implying that holomorphic maps from open Riemann surfaces to $\mathrm{SL}_{2}(\mathbb{C})$ and elements of $\mathrm{SL}_{2}(\mathcal{A})$ are null-homotopic. This is the reason that the map in question being null-homotopic is a redundant assumption in Theorem 1 and 2. As corollaries of Theorem 1 and 2 we get the analogous results if the special linear group is replaced by the linear group with the corresponding entries.

Corollary 1. Any null-homotopic holomorphic map from an open Riemann surface to the linear group $\mathrm{GL}_{2}(\mathbb{C})$ is a product of two exponentials.

Proof. Let $X$ be an open Riemann surface and $\mathrm{M}_{2}(\mathbb{C})$ the complex $2 \times 2$ matrices. If a given holomorphic map $A: X \rightarrow \mathrm{GL}_{2}(\mathbb{C})$ is null-homotopic, then $\operatorname{det} A: X \rightarrow \mathbb{C}^{*}$ is null-homotopic as well. Therefore $\operatorname{det} A$ has a holomorphic logarithm $\log : X \rightarrow \mathbb{C}$, satisfying $e^{\log }=\operatorname{det} A$. In particular, if $D: X \rightarrow \mathrm{M}_{2}(\mathbb{C})$ is the diagonal matrix with diagonal entries $\log / 2$, $\exp (-D) A$ has values in $\mathrm{SL}_{2}(\mathbb{C})$. By Theorem 1 there are holomorphic $B, C: X \rightarrow \mathrm{M}_{2}(\mathbb{C})$ such that

$$
A=e^{D} e^{-D} A=e^{D} e^{B} e^{C}=e^{D+B} e^{C},
$$

where we used in the last equality that $D$ commutes with all other matrices. This finishes the proof.

Unlike in Theorem 1 , in Corollary 1 the assumption that $f$ is null-homotopic is not redundant. For instance,

$$
A(z)=\left(\begin{array}{cc}
z & 0 \\
0 & z
\end{array}\right), z \in \mathbb{C}^{*}
$$

is not null-homotopic since otherwise $\operatorname{det} A: \mathbb{C}^{*} \rightarrow \mathbb{C}^{*}, z \mapsto z^{2}$ would be null-homotopic as well.

Corollary 2. For the disc algebra $\mathcal{A}$, any element of $\mathrm{GL}_{2}(\mathcal{A})$ is a product of two exponentials.

Proof. This follows from Theorem 2 in the same way as Corollary 1 follows from Theorem 1. Here, we need in addition that any unit in $\mathcal{A}$ has a logarithm, which follows from the fact that the disc (and thereby the domain of the elements of $\mathcal{A}$ ) is contractible. In particular, the map in question being null-homotopic is again a redundant assumption.

Corollary 2 improves a result of Mortini and Rupp, who showed the same with four instead of two factors in the conclusion, see Theorem 7.1 in [8]. Also Corollary 1 and 2 are sharp in the sense that one exponential factor is not enough. An example is the matrix

$$
A(z)=\left(\begin{array}{cc}
1 & 1 \\
0 & e^{4 \pi i z}
\end{array}\right), z \in \Delta .
$$

One can show that the second entry of any lift of $z \mapsto A(z),|z|<1 / 2$ via the exponential map tends to infinity if $z \rightarrow 1 / 2$. For details see [8], Example 6.4.

We would like to thank Sebastian Baader for helpful comments on a draft of this text. 


\section{Proof of Theorem 1}

An important ingredient in the proof is an Oka principle due to Forstnerič, which follows essentially from Theorem 2.1 in [2]. The version, which we use in this text is the below stated Theorem 3. It is used to show Proposition 1. which is the main ingredient in the proof of Theorem 1. Throughout this section $X$ denotes an open Riemann surface.

Proposition 1. Let $A: X \rightarrow \mathrm{SL}_{2}(\mathbb{C})$ be holomorphic and assume that $A(x)$ has distinct eigenvalues for some $x \in X$. Then $A=B C$ for suitable holomorphic $B, C: X \rightarrow \mathrm{SL}_{2}(\mathbb{C})$, both of which have vanishing trace.

Note that the conclusion of Proposition 1 is equivalent to finding a holomorphic $B: X \rightarrow \mathrm{SL}_{2}(\mathbb{C})$ such that $B$ and $A B$ have vanishing trace, simply since taking the inverse of a $2 \times 2$-matrix with trace zero has again trace zero. Expressed differently, Proposition 1 is proved if we can show the existence of a global section of the bundle

$$
Z:=\left\{(x, B) \in X \times \mathrm{SL}_{2}(\mathbb{C}): \operatorname{tr}(B)=\operatorname{tr}(A(x) B)=0\right\}
$$

over $X$. If $a, b, c, d$ denote the coefficients of $A$, and $u, w, v,-u$ denote the coefficients of $B$, we can express $Z$ more explicitly as

$\left\{(x, u, v, w) \in X \times \mathbb{C}^{3}:(a(x)-d(x)) u+b(x) v+c(x) w=0, u^{2}+v w=-1\right\}$.

More concretely, Proposition 1 is proved if we manage the prove the following reformulation.

Proposition 2. Let $A: X \rightarrow \mathrm{SL}_{2}(\mathbb{C})$ be holomorphic and assume that $A(x)$ has distinct eigenvalues for some $x \in X$. Then the restriction $h$ of the projection $X \times \mathbb{C}^{3} \rightarrow X$ to $Z$ has a holomorphic section.

For an open subset $U \subset X, Z \mid U$ denotes the restriction of the bundle $h: Z \rightarrow X$ to $h^{-1}(U)$. We start the proof of Proposition 2 with the following simple

Lemma 1. For every $x \in X$ there is a neighborhood $U$ of $x$ and a holomorphic section $F: U \rightarrow Z \mid U$ of $Z \mid U$.

Proof. After passing to a local chart we may assume that $X$ is the unit disc $\Delta:=\{z \in \mathbb{C}:|z|<1\}$ and $x=0$. Finding a local holomorphic section in a neighborhood of 0 is equivalent to finding a neighborhood $0 \in U \subset \Delta$ and holomorphic maps $u, v, w: U \rightarrow \mathbb{C}$, which satisfy

$$
(a-d) u+b v+c w=0, \quad u^{2}+v w=-1 .
$$

Local holomorphic solutions to (1) exist if and only if there are local holomorphic solutions to the less restrictive problem

$$
(a-d) u+b v+c w=0, \quad u^{2}+v w \in \mathcal{O}_{0}^{*} .
$$

The reason is that if $u, v, w$ are local solutions in a neighborhood of the origin to (2), we can rescale these solutions with a local holomorphic square root of $u^{2}+v w$, or more precisely, by defining new solutions by $\frac{i u}{r}, \frac{i v}{r}, \frac{i w}{r}$ for some $r: U \rightarrow \mathbb{C}^{*}$ satisfying $r^{2}=u^{2}+v w$ defined on a sufficiently small neighborhood $U$ of the origin. To find solutions to (2) we distinguish three cases. Let $n(f) \in \mathbb{Z}_{\geq 0}$ denote the vanishing order of a holomorphic function 
$f: \Delta \rightarrow \mathbb{C}$ at the origin. The first case is $n(a-d) \geq n(b)$. Then $-\frac{a-d}{b}$ is holomorphic in a neighborhood of 0 and $u=1, v=-\frac{a-d}{b}$ and $w=0$ is a solution to (2). The second case $n(a-d) \geq n(c)$ we find similarly a solution $u=1, v=0$ and $w=-\frac{a-d}{c}$ to (2). The remaining case is $n(a-d)<\min (n(b), n(c))$, which implies $n(a-d)<n(b+c)$ and hence $-\frac{b+c}{a-d}$ is holomorphic in a neighborhood of the origin and vanishes at the origin. Then $u=-\frac{b+c}{a-d}, v=1, w=1$ solves (2). This finishes the proof.

Let $D$ denote the discriminant of $A$, that is $D:=(a+d)^{2}-4$. By isomorphic fiber bundles we mean isomorphic as complex analytic fiber bundles.

Lemma 2. Let $U \subset X \backslash(\{D=0\} \cup\{c=0\})$ be an open neighborhood where $D: U \rightarrow \mathbb{C}$ has a holomorphic square root $\sqrt{D}$, and set $f:=\frac{d-a+\sqrt{D}}{2 c}$. Then $Z \mid U$ is isomorphic to $U \times \mathbb{C}^{*}$, and an isomorphism is given by

$$
\phi: Z \mid U \rightarrow U \times \mathbb{C}^{*}, \phi(x, u, v, w)=(x, u+f(x) v) .
$$

Proof. First we do the necessary computations at the level of a single fiber. For this, we think of the coefficients $a, b, c, d$ of $A$ as elements of $\mathbb{C}$. We want to determine all $u, v, w \in \mathbb{C}$ such that

$$
(a-d) u+b v+c w=0, \quad-u^{2}-v w=1 .
$$

Since $c \neq 0$, we can solve for $w$ and get equivalently

$$
\begin{aligned}
-1 & =u^{2}+v w \\
& =u^{2}+v \frac{(d-a) u-b v}{c} \\
& =u^{2}+\frac{d-a}{c} u v-\frac{b}{c} v^{2} \\
& =\left(u+\frac{d-a}{2 c} v\right)^{2}-\left(\frac{(d-a)^{2}}{4 c^{2}}+\frac{b}{c}\right) v^{2} .
\end{aligned}
$$

Furthermore we have

$$
\frac{(d-a)^{2}}{4 c^{2}}+\frac{b}{c}=\frac{(d+a)^{2}-4 a d}{4 c^{2}}+\frac{4 b c}{4 c^{2}}=\frac{(d+a)^{2}-4(a d-b c)}{4 c^{2}}=\frac{D}{4 c^{2}} .
$$

Fix a square root $\sqrt{D}$ of $D$ and note that

$$
\tilde{u}=u+\frac{d-a+\sqrt{D}}{2 c} v, \quad \tilde{v}=u+\frac{d-a-\sqrt{D}}{2 c} v
$$

defines a linear coordinate change of $\mathbb{C}^{2}$, which translates the above equation to

$$
\begin{aligned}
-1 & =\left(u+\frac{d-a}{2 c} v\right)^{2}-\frac{D}{4 c^{2}} v^{2} \\
& =\left(u+\frac{d-a}{2 c} v\right)^{2}-\left(\frac{\sqrt{D}}{2 c} v\right)^{2} \\
& =\left(u+\frac{d-a+\sqrt{D}}{2 c} v\right)\left(u+\frac{d-a-\sqrt{D}}{2 c} v\right) \\
& =\tilde{u} \tilde{v} .
\end{aligned}
$$

This shows that the fiber is given by $\left\{(\tilde{u}, \tilde{v}) \in \mathbb{C}^{2}: \tilde{u} \tilde{v}=-1\right\}=\mathbb{C}^{*}$ and that $(u, v, w) \rightarrow u+\frac{d-a+\sqrt{D}}{2 c} v$ is an isomorphism of the fiber onto $\mathbb{C}^{*}$. Moreover, our computations yield a trivialization of $Z \mid U$, which is defined similarly, or more precisely, as in the assumption of the Lemma. This is the case since 
our computations work out just the same way if we have a holomorphic dependence on $x \in U$.

Lemma 3. Over $X \backslash\{D=0\}, h: Z \rightarrow X$ is a fiber bundle with fiber $\mathbb{C}^{*}$.

Proof. At points $x \in X \backslash\{D=0\}$ with $c(x) \neq 0$, choose a neighborhood $U \subset X$ of $x$ such that $c \mid U$ does not vanish, and such that $D$ has a square root on $U$. Then a trivialization of $Z \mid U$ is given by Lemma 2 . In the case $c(x)=0$, let us reduce the problem to the case $c(x) \neq 0$ with the following observation. Our bundle is given by

$$
Z=\left\{(x, B) \in X \times \mathrm{SL}_{2}(\mathbb{C}): \operatorname{tr}(B)=\operatorname{tr}(A(x) B)=0\right\} .
$$

Define for $P \in \mathrm{SL}_{2}(\mathbb{C})$ a bundle

$$
Z_{P}=\left\{\left(x, P B P^{-1}\right) \in X \times \mathrm{SL}_{2}(\mathbb{C}): \operatorname{tr}(B)=\operatorname{tr}(A(x) B)=0\right\} .
$$

Clearly $Z$ and $Z_{P}$ are isomorphic over $X$. Since conjugation with a matrix does not change the trace, we obtain with the substitution $C=P B P^{-1}$

$$
\begin{aligned}
Z_{P} & =\left\{(x, C) \in X \times \mathrm{SL}_{2}(\mathbb{C}): \operatorname{tr}\left(P^{-1} C P\right)=\operatorname{tr}\left(A(x) P^{-1} C P\right)=0\right\} \\
& =\left\{(x, C) \in X \times \mathrm{SL}_{2}(\mathbb{C}): \operatorname{tr}(C)=\operatorname{tr}\left(P A(x) P^{-1} C\right)=0\right\} .
\end{aligned}
$$

Note that if the third entry $c$ of $A$ equals 0 at $x$, then, since $D(x) \neq 0$ and hence $A(x) \neq \pm i d$, there is $P \in \mathrm{SL}_{2}(\mathbb{C})$ such that the third entry of $P A(x) P^{-1}$ does not vanish. Using that $Z$ and $Z_{P}$ are isomorphic and that we can solve the problem for $Z_{P}$ close to $x$, the statement follows.

To finish the proof of Propostion 2 we need the following special case of Theorem 6.14.6, p. 310 in 3 .

Theorem 3. Let $h: Z \rightarrow X$ be a holomorphic map of a reduced complex space $Z$ onto a reduced Stein space $X$. Let $X^{\prime} \subset X$ be a complex analytic subvariety and let $Z^{\prime}:=h^{-1}\left(X^{\prime}\right)$ and assume that the restriction $h: Z \backslash$ $Z^{\prime} \rightarrow X \backslash X^{\prime}$ is an elliptic submersion. Moreover, let $f: X \rightarrow Z$ be a continuous section of $h$ which is holomorphic in a neighborhood of $X^{\prime}$. Then $f$ is homotopic through continuous sections of $h$ which are holomorphic in a fixed small neighborhood of $X^{\prime}$ to a holomorphic section of $h$.

A consequence of this is the following

Proposition 3. Let $h: Z \rightarrow X$ be a holomorphic map from a reduced complex space onto an open Riemann surface. Moreover, assume that there is a discrete set $X^{\prime} \subset X$ such that for $Z^{\prime}=h^{-1}\left(X^{\prime}\right)$, the restriction $h$ : $Z \backslash Z^{\prime} \rightarrow X \backslash X^{\prime}$ is a fiber bundle with fiber $\mathbb{C}^{*}$ and assume that there is a local holomorphic section in a neighborhood of every point of $X^{\prime}$. Then $h$ has a global holomorphic section $f: X \rightarrow Z$.

Proof. First we show the existence of a continuous section which is holomorphic in a neighborhood $U$ of $X^{\prime}$. By assumption there is a local holomorphic section $f: U \rightarrow Z$ of $h$ defined on a neighborhood $U$ of $X^{\prime}$. By possibly shrinking $U$ we may assume that every connected component of $U$ contains exactly one point of $X^{\prime}$ and is homeomorphic to a disc, and that $f$ extends continuously to $\bar{U} . \quad X \backslash X^{\prime}$ is an open Riemann surface and thus deformation retracts onto a 1-dimensional CW-complex $K$, see e.g. [4. After possibly modifying a fixed deformation retract $r$ of $X \backslash X^{\prime}$ onto $K$ by a 
conjugation with a suitable homeomorphism of $X \backslash X^{\prime}$ we can assume that $\partial U \subset K$. Since the fiber $\mathbb{C}^{*}$ of $Z$ is connected we can extend $f \mid \partial U$ to a section $\tilde{f}: K \rightarrow Z \mid K$. Since $K$ is a deformation retract of $X \backslash X^{\prime}$ and $h: Z \backslash Z^{\prime} \rightarrow X \backslash X^{\prime}$ is a fiber bundle, the section $\tilde{f}$ extends to a continuous section $F: X \backslash X^{\prime} \rightarrow Z \backslash Z^{\prime}$, see e.g. Theorem 7.1, p. 21 in [5]. Since $f$ and $F \mid X \backslash U$ agree on $\partial U$, these two sections define a continuous section $X \rightarrow Z$ which agrees with the holomorphic section $f$ on the neighborhood $U$ of $X^{\prime}$. The existence of a global holomorphic section follows now from the above Oka principle due to Forstnerič, see Theorem 3, This finishes the proof.

Proof of Proposition Q Let $h: Z \rightarrow X$ be the bundle over $X$ from Proposition 2. With Lemma 1 we proved that there are local sections of $h$ at every point $x \in X$, in particular also at points of the discrete set $X^{\prime}=\{D=0\}$. Moreover, with Lemma 3 we showed that $h$ is a locally trivial $\mathbb{C}^{*}$-bundle over $X \backslash\{D=0\}$. It follows now from Proposition 3 that there is a holomorphic section of $h$. This finishes the proof.

Lemma 4. Let $X$ be an open Riemann surface and let $A: X \rightarrow \mathrm{SL}_{2}(\mathbb{C})$ be holomorphic with vanishing trace. Then $A=e^{B}$ for some holomorphic $B: X \rightarrow \mathrm{M}_{2}(\mathbb{C})$ with vanishing trace.

Proof. The characteristic polynomial of $A$ equals $T^{2}+1$. In particular $\pm i$ are the eigenvalues (at every point $x \in X$ ). There are line bundles $E(i)$ and $E(-i)$ over $X$, whose non-vanishing sections correspond to holomorphic eigenvectors of $i$ and $-i$ respectively. Explicitly, we have

$$
\begin{aligned}
E(i) & :=\left\{(x, z) \in X \times \mathbb{C}^{2}: A(x) z=i z\right\}, \\
E(-i) & :=\left\{(x, z) \in X \times \mathbb{C}^{2}: A(x) z=-i z\right\} .
\end{aligned}
$$

Since every line bundle over an open Riemann surface is trivial, we have $E(i) \cong X \times \mathbb{C} \cong E(-i)$ as complex analytic line bundles. This implies that there are two holomorphic eigenvectors $v: X \rightarrow E(i), w: X \rightarrow E(-i)$ with $v(x) \neq 0 \neq w(x)$ for all $x \in X$. In particular

$$
P: X \rightarrow \mathrm{M}_{2}(\mathbb{C}), P(x):=(v(x) w(x))
$$

takes values in $\mathrm{GL}_{2}(\mathbb{C})$ since $v(x)$ and $w(x)$ are eigenvectors of $A(x)$ to the distinct eigenvalues $\pm i$. This implies that $A$ is holomorphically diagonalisable with

$$
A=P D P^{-1}, \quad D:=\left(\begin{array}{cc}
i & 0 \\
0 & -i
\end{array}\right) .
$$

For the diagonal matrix $\tilde{D}$ with entries $\pm \frac{i \pi}{2}$ we have $e^{\tilde{D}}=D$. We get for $B:=P \tilde{D} P^{-1}$ the equality

$$
A=P D P^{-1}=P e^{\tilde{D}} P^{-1}=e^{P \tilde{D} P^{-1}}=e^{B},
$$

as desired. Note that $B$ has vanishing trace since $\tilde{D}$ has vanishing trace. This finishes the proof.

Proof of Theorem 1. Let $X$ be an open Riemann surface and let $A: X \rightarrow$ $\mathrm{SL}_{2}(\mathbb{C})$ be a holomorphic map. If the characteristic polynomial of $A$ equals $(T-1)^{2}$, then, since $(A-i d)^{2}=\chi_{A}(A)=0$ by Cayley-Hamilton, we have

$$
\exp (A-i d)=i d+(A-i d)=A \text {. }
$$


Moreover, the trace of $A$ is equal to minus the second coefficient of the characteristic polynomial, which implies in our case that $\operatorname{tr}(A-i d)=0$, as desired. This shows that $A$ can be written as a single exponential factor. If the characteristic polynomial is $(T+1)^{2}$, then the characteristic polynomial of $-A$ is $(T-1)^{2}$ and since $-i d$ is equal to the exponential of the diagonal matrix with diagonal entries $\pi i$ and $-\pi i, A$ is a product of at most two exponentials with vanishing trace. Otherwise there is $x \in X$ such that $A(x)$ has distinct eigenvalues. In that case it follows from Proposition 1 that $A=B C$ for holomorphic $B, C: X \rightarrow \mathrm{SL}_{2}(\mathbb{C})$ with vanishing trace. In particular, the characteristic polynomials of $B$ and $C$ are both $(T-i)(T+i)$. Since $B$ and $C$ have a logarithm by Lemma 4 , we are done.

\section{Proof of Theorem 2}

The proof depends essentially on three ingredients. The first ingredient is that the Bass stable rank of the disc algebra $\mathcal{A}$ equals 1 . This is needed to reduce the problem to matrices with an invertible first entry. The second and third ingredient are the simple facts that the elements of $\mathcal{A}$ are bounded, and that $\exp : \mathcal{A} \rightarrow \mathcal{A}$ is onto to units of $\mathcal{A}$. In the following $\bar{\Delta} \subset \mathbb{C}$ denotes the closed unit disc centered at the origin. We use the following notation. If $f: \bar{\Delta} \rightarrow \mathbb{C}$ is a function, then $|f|: \bar{\Delta} \rightarrow \mathbb{R}$ denotes the absolute value $z \mapsto|f(z)|$. In particular, the symbol $|f|$ should not be confused with the sup-norm on $\mathcal{A}$, which is not used explicitly in the proof. Moreover, for $f, g: \bar{\Delta} \rightarrow \mathbb{R}$ we write $f>g$ if $f(z)>g(z)$ for all $z \in \bar{\Delta}$. The proof depends on the following elementary lemma.

Lemma 5. Let $f \in \mathcal{A}$ be such that $|f|>2$. Then the polynomial $T^{2}-f T+1$ has roots $\lambda, \lambda^{-1} \in \mathcal{A}$ such that $|\lambda|>1$.

Proof. First note that our assumption implies that the discriminant $f^{2}-4$ does not vanish. Therefore $f^{2}-4$ has a square root in $\mathcal{A}$, which implies that there are roots $\lambda, \lambda^{-1} \in \mathcal{A}$ of $T^{2}-f T+1$. We have to show that one of $|\lambda|$ and $\left|\lambda^{-1}\right|$ is strictly larger than 1 . Note that if $T^{2}-z T+1, z \in \mathbb{C}$ has a root $r \in \mathbb{C}$ with $|r|=1$, then we get $|z|=\left|r^{2}+1\right| /|r|=\left|r^{2}+1\right| \leq 2$. Expressed differently, if $|z|>2$, then $T^{2}-z T+1$ has no root on the unit circle. This implies that $\lambda$ and $\lambda^{-1}$ avoid the unit circle, and moreover - by continuity of $\lambda$ and $\lambda^{-1}$ - that exactly one of the two is strictly bigger than 1 in absolute value.

Proof of Theorem 国. Let

$$
A=\left(\begin{array}{ll}
a & b \\
c & d
\end{array}\right) \in \operatorname{SL}_{2}(\mathcal{A}) .
$$

It is well-known that the Bass stable rank of $\mathcal{A}$ equals 1 , see [7]. By definition of the Bass stable rank this means that for any pair $f, g \in \mathcal{A}$ with $f \mathcal{A}+g \mathcal{A}=$ $\mathcal{A}$, there is $h \in \mathcal{A}$ such that $f+h g$ is a unit in $\mathcal{A}$. In particular, since $a d-b c=1$, there is $h \in \mathcal{A}$ such that $a+h c=1$. Consequently the first entry of

$$
\left(\begin{array}{ll}
1 & h \\
0 & 1
\end{array}\right)\left(\begin{array}{ll}
a & b \\
c & d
\end{array}\right)\left(\begin{array}{cc}
1 & -h \\
0 & 1
\end{array}\right)=\left(\begin{array}{cc}
a+h c & * \\
* & *
\end{array}\right)
$$


is a unit. Since conjugation with matrices in $\operatorname{GL}_{2}(\mathcal{A})$ does not change the number of needed exponential factors to represent a given matrix, this shows that it suffices to consider the case where the first entry $a$ of $A$ is a unit. For such $A$, the strategy is as follows: for $\delta>0$ set

$$
B:=\left(\begin{array}{cc}
\delta & 0 \\
0 & 1 / \delta
\end{array}\right)\left(\begin{array}{ll}
a & b \\
c & d
\end{array}\right)=\left(\begin{array}{cc}
\delta a & \delta b \\
c / \delta & d / \delta
\end{array}\right) \in \mathrm{SL}_{2}(\mathcal{A}) .
$$

If we find $\delta$ such that $B=B(\delta)$ has a logarithm, then - since $A$ is the product of the diagonal matrix with entries $1 / \delta, 0,0, \delta$ and $B$ - we know that $A$ is a product of two exponentials. Our claim is that $B$ has a logarithm for any sufficiently large $\delta>0$. To see this, let $\delta \geq 1$ be an upper bound of the (bounded) function

$$
\beta=\frac{3+|d|}{|a|} .
$$

From the fact that $\delta \geq 1$ is an upper bound of $\beta$ it follows that

$$
|\operatorname{tr}(B)|=|\delta a+d / \delta| \geq \delta|a|-\frac{|d|}{\delta} \geq(3+|d|)-|d|>2 .
$$

By Lemma 5 we know that the characteristic polynomial $\chi_{B}=T^{2}-\operatorname{tr}(B) T+$ 1 has roots $\lambda, \lambda^{-1} \in \mathcal{A}$ with $|\lambda|>1$. Since $\lambda$ is a unit in $\mathcal{A}$, the matrix $D$ with diagonal entries $\lambda$ and $\lambda^{-1}$ has a logarithm given by the diagonal matrix with diagonal entries $\log (\lambda) \in \mathcal{A}$ and $-\log (\lambda) \in \mathcal{A}$ for some fixed logarithm of $\lambda$. Moreover, since conjugation with an element in $\mathrm{GL}_{2}(\mathcal{A})$ does not change the number of needed exponential factors, it suffices to find $P \in \mathrm{GL}_{2}(\mathcal{A})$ with

$$
B=P D P^{-1} \text {. }
$$

Our claim is that

$$
P=\left(\begin{array}{cc}
d / \delta-\lambda & -\delta b \\
-c / \delta & \delta a-\lambda^{-1}
\end{array}\right) \in \mathrm{M}_{2}(\mathcal{A})
$$

does the job. To show this it suffices to show that the columns $v$ resp. $w$ of $P=(v w)$ satisfy $(B-\lambda i d) v=\left(B-\lambda^{-1} i d\right) w=0$ and that $|\operatorname{det} B| \geq 1$. For the first part we get

$$
(B-\lambda i d) v=\left(\begin{array}{cc}
\delta a-\lambda & \delta b \\
c / \delta & d / \delta-\lambda
\end{array}\right)\left(\begin{array}{c}
d / \delta-\lambda \\
-c / \delta
\end{array}\right)=\left(\begin{array}{c}
\chi_{B}(\lambda) \\
0
\end{array}\right)=0,
$$

and similarly

$$
\left(B-\lambda^{-1} i d\right) w=\left(\begin{array}{cc}
\delta a-\lambda^{-1} & \delta b \\
c / \delta & d / \delta-\lambda^{-1}
\end{array}\right)\left(\begin{array}{c}
-\delta b \\
\delta a-\lambda^{-1}
\end{array}\right)=\left(\begin{array}{c}
0 \\
\chi_{B}\left(\lambda^{-1}\right)
\end{array}\right)=0 .
$$

For the second part, we get with $a d-b c=1$

$$
\operatorname{det} P=-\delta \lambda a-\delta^{-1} \lambda^{-1} d+2 .
$$

It follows from $|\lambda|>1$ that

$$
|\operatorname{det} P| \geq \delta|\lambda||a|-\delta^{-1}\left|\lambda^{-1}\right||d|-2 \geq \delta|a|-\delta^{-1}|d|-2 .
$$

Furthermore, the fact that $\delta \geq 1$ bounds $\beta=(3+|d|) /|a|$ from above yields

$$
\delta|a|-\delta^{-1}|d|-2 \geq(3+|d|)-|d|-2=1,
$$

which shows that $|\operatorname{det} P| \geq 1$. This finishes the proof. 


\section{REFERENCES}

[1] E. Doubtsov, F. Kutzschebauch: F. Anal. Math. Phys. (2019). https://doi.org/10.1007/s13324-019-00289-8

[2] F. Forstnerič: The Oka principle for multivalued sections of ramified mappings. Forum Math. 15(2), 309-328 (2003)

[3] F. Forstnerič: Stein Manifolds and Holomorphic Mappings (The Homotopy Principle in Complex Analysis, Second Edition), Ergebnisse der Mathematik und ihrer Grenzgebiete, 3. Folge, Springer-Verlag, Berlin Heidelberg (2017)

[4] H. Hamm: Deformation retracts of Stein spaces, Math. Ann. 308(2), 333-345 (1997)

[5] D. Husemoller: Fibre bundles, Graduate Texts in Mathematics 20, Third Edition, Springer-Verlag, New York (1994)

[6] B. Ivarsson, F. Kutzschebauch: Holomorphic factorization of mappings into $\mathrm{SL}_{2}(\mathbb{C})$, Ann. Math. (2) 175(1), 45-69 (2012)

[7] P. W. Jones, D. Marshall, T. H. Wolff: Stable rank of the disc algebra, Proc. Amer. Math. Soc. 96, 603-604 (1986)

[8] R. Mortini, R. Rupp: Logarithms and Exponentials in the Matrix Algebra $\mathcal{M}_{2}(A)$, Comput. Methods Funct. Theory 18, 53-87 (2018)

E-mail address: frank.kutzschebauch@math.unibe.ch, luca.studer@math.unibe.ch 